Special issue of the International Conference on Computational and Experimental Science and Engineering (ICCESEN 2014)

\title{
Variation of Energy Resolution with Distance for a $\mathrm{NaI}(\mathrm{Tl})$ Detector
}

\author{
İ. Akkurt, S.S. Arda*, K. Gunoglu \\ Süleyman Demirel University, Physics Department, Isparta, Turkey
}

As the nuclear radiation has started to be used in a variety of different fields, it is important to be protected from it, and thus the radiation measurement becomes vital. The quality of the performance of a detection system, used for the energy measurements, is important. It is characterized by the width of the pulse-height distribution, obtained for the particles of the same energy (monoenergetic source). The energy spectrum of a radiation source depends on the type and energy of the incident particle and the type of the detector. In this work the energy resolution of a $3^{\prime \prime} \times 3^{\prime \prime} \mathrm{NaI}(\mathrm{Tl})$ detector has been measured for photon energies of 511, 662, 835, 1173, 1275, and $1332 \mathrm{keV}$, and its variation with the detector-source distance was investigated. The energy resolution of a detector system is obtained from the peak full width at one-half of the maximum height (FWHM) of a single peak (for a particular energy) as a function of detector-source distance. It was found that the energy resolution has decreased with the increasing distance.

DOI: 10.12693/APhysPolA.128.B-422

PACS: 29.40.-n, 29.30.Kv

\section{Introduction}

As the nuclear radiation is getting used in a variety of different fields, its measurement becomes important. The quality of the performance of a detection system used for energy measurements is also important. It is characterized by the width of the pulse-height distribution, obtained with particles of the same energy (monoenergetic source). The energy spectrum of a radiation source depends on the type and energy of the incident particle and the type of the detector. An important aspect of an energy spectrum is the ability to distinguish between gamma rays with slightly different energy. This is the so called energy resolution, which is defined as the full width at half maximum (FWHM) of the photo peak at a certain energy.

In this work energy, the energy resolution of a $3^{\prime \prime} \times 3^{\prime \prime}$ $\mathrm{NaI}(\mathrm{Tl})$ detector has been measured for photon energies of 511,662, 1173, 1275, and $1332 \mathrm{keV}$ and its variation with the detector-source distances was investigated.

\section{Materials and methods}

The energy resolution has been measured using radioactive sources and a $\mathrm{NaI}(\mathrm{Tl})$ detector $3^{\prime \prime} \times 3^{\prime \prime}$ in size.
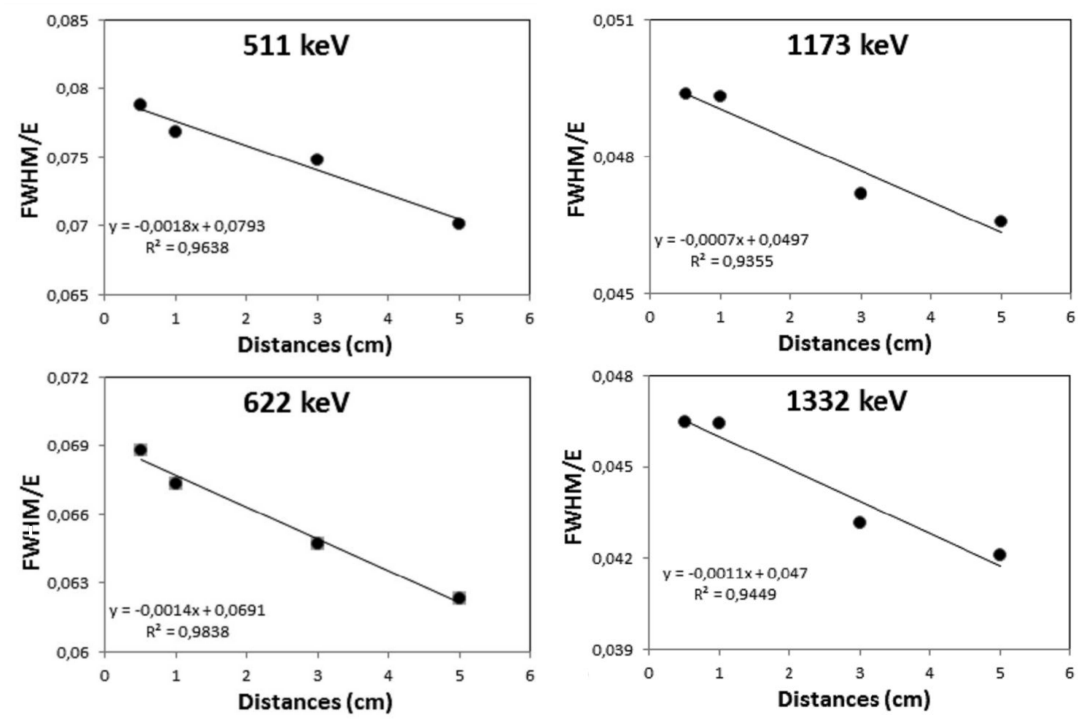

Fig. 1. Variation of energy resolution as a function of distance, for gamma ray energies of 511, 622, 1173 and $1332 \mathrm{keV}$.

*corresponding author; e-mail: sule.arda@hotmail.com 


\section{Results and discussion}

The energy resolution of $\mathrm{NaI}(\mathrm{Tl})$ detector has been measured at four different distances from the radiation source at gamma ray energies of 511, 662, 1173 and $1332 \mathrm{keV}$. The results are presented in Fig. 1. It can be seen, that the energy resolution decreases with the increasing detector-source distance.

\section{Acknowledgments}

This work is supported by Suleyman Demirel University (3312-YL2-12) and State Planning Unit of Turkey (Grant No: DPT2006K-120470).

\section{References}

[1] I. Akkurt, H. Akyıldırım, B. Mavi, S. Kılınçarslan, C. Basyigit, Annals of Nucl. Energy 37, 910 (2010).

[2] I. Akkurt, H. Akyıldırım, Nucl. Eng. Des. 252, 163 (2012).

[3] B. Mavi, I. Akkurt, Radiat. Phys. Chem. 79, 933 (2010). 